\section{Desigualdad socioeconómica y salud mental: revisión de la literatura latinoamericana}

\author{
Socioeconomic inequality and mental health: \\ a Latin American literature review
}

\author{
${ }^{1}$ Departamento en Atención \\ a la Salud, Universidad \\ Autónoma Metropolitana \\ Xochimilco, México DF, \\ México. \\ 2 Instituto Nacional de \\ Psiquiatría Ramón de la \\ Fuente Muñiz, Secretaría de \\ Salud, México DF, México. \\ Correspondencia \\ L. Ortiz-Hernández \\ Departamento de Atención \\ a la Salud, Universidad \\ Autónoma Metropolitana \\ Xochimilco. \\ Calzada del Hueso 1100 \\ Col. Villa Quietud, Coyoacán, \\ México, DF 04960 \\ lortiz@correo.xoc.uam.mx
}

\begin{abstract}
This study provides a review of the scientific output in Latin America concerning the impact of socioeconomic status (SES) on mental disorders and drug use or addiction. International and regional databases were analyzed. According to the majority of the studies, adults and adolescents with low SES showed increased risk of mental disorders, and alcohol consumption was higher among individuals with high SES, while low SES was associated with alcohol abuse and addiction, although the evidence was less conclusive. Smoking was more frequent among young people with high SES, but in adults it was more common with low SES. Illicit drug use was more frequent among adults (but not adolescents) with low SES. Prescription drugs tended to be consumed by adults and adolescents with higher SES. Use of solvents was more frequent among low SES adolescents. The studies' observed trends and methodological aspects are also discussed.
\end{abstract}

Substance-Related Disorders; Psychological Stress; Mental Disorders; Socioeconomic Factors
Luis Ortiz-Hernández 1

Sergio López-Moreno 1

Guilherme Borges 1,2

\section{Introducción}

Diversas fuentes muestran que en América Latina los problemas relacionados con la salud mental deben ser motivo de atención por su frecuencia y la discapacidad a la que se asocian. A finales de la década de los ochenta 1 en América Latina entre $4,4 \%$ y $24,4 \%$ de los varones presentaban alcoholismo y la prevalencia de fármaco-dependencia oscilaba entre $1,1 \%$ y $7,7 \%$; mientras que el porcentaje de años de vida potencial perdidos por muertes violentas (homicidio y suicidio) variaron entre 5,9\% y 21,4\%. En 1990 en América Latina y el Caribe el 15,9\% de los años de vida perdidos ajustados por discapacidad se debieron a trastornos neuropsiquiátricos 2; dicha proporción fue menor a la de otras dos regiones (economías de mercado establecidas y países europeos que eran socialistas), pero mayor a la de otras cinco áreas del mundo (China, el resto de Asía, Medio Oriente, India y África subsahariana). Para el año $2000^{3}$, los trastornos depresivos unipolares contribuyeron con el 8\% de los DALYs en América, ocupando la primera posición, lo cual contrasta con otras regiones donde esos trastornos ocuparon los segundos lugares (Pacífico occidental), tercero, (Europa), cuarto (Sureste asiático), quinto (Este del Mediterráneo) o no aparecieron en las primeras diez causas (África). Entre 1995 y 2002 la tasa suicidios se incrementó en Argentina (de 6,6 a 8,2 casos por 100,000 habitantes), Costa Rica (de 5,4 a 6,7), México (de 
3,4 a 4,1), Nicaragua (de 9,1 a 13,2) y Panamá (de $5,6$ a 6,3$)^{4}$.

Considerando los indicadores clásicos de salud poblacional, es posible verificar que en América la desigualdad social en salud existe tanto entre países como en el interior de los mismos. Al considerar como unidad de análisis los países de la región, la tasa de mortalidad infantil se relaciona positivamente con el producto interno bruto per cápita y el porcentaje de la población con acceso a agua potable 5 . Respecto a las disparidades en el interior de las sociedades, al comparar las tasas de mortalidad infantil de las regiones subnacionales (estados, departamentos o provincias) de 19 países de América se observó que las razones de mortalidad iban de 1,67 (en Chile) a 6,22 (en Colombia); en 16 países las razones de mortalidad infantil entre las regiones fueron mayores a 26 .

América Latina es la región con mayor polarización socioeconómica, es decir, donde la riqueza está más concentrada en una menor proporción de la población. En la década de los noventa la media de los coeficientes de Gini de los países latinoamericanos fue de 0,555, superando la de Europa oriental $(0,465)$, África $(0,521)$, Estados Unidos de Norteamérica, Europa occidental y Oceanía $(0,394)$; el único continente que presentó mayor desigualdad fue Asia $(0,615){ }^{7}$. Además, la inequidad socioeconómica se incrementó en los últimos años, lo que ha sido paralelo al aumento de la población en situación de pobreza. Entre 1990 y 1999, en ocho de catorce países latinoamericanos aumentó el porcentaje del ingreso captado por el $10 \%$ de las familias de mayor ingreso, mientras que sólo en dos países esa proporción disminuyó y en los cuatro restantes se mantuvo sin cambios 8. Entre 1980 y 1990 en América Latina la proporción de pobres pasó de $34,7 \%$ a $41 \%$, para luego descender a $35,3 \%$ en 1999; en el caso de las zonas urbanas las cifras fueron $25,3 \%$, 35\% y 29,8\%, respectivamente 8 .

Los rasgos socioeconómicos de América Latina y los indicios de que en la región los trastornos mentales cada vez cobrarán mayor relevancia hacen ver que es relevante estudiar el vínculo entre posición socioeconómica y salud mental. Para estudiar la relación de las condiciones socioeconómicas con los daños a la salud es necesario remitirse al concepto de desigualdad social en salud.

\section{La desigualdad social en salud}

"La práctica de la medicina siempre ha estado relacionada con las condiciones sociales y económicas de grupos específicos de personas, pero estas relaciones sólo rara vez fueron objeto de discusión teórica. Sólo hasta la época moderna aparece una clara conciencia de los estrechos lazos que existen entre las condiciones sociales y los problemas médicos" 9 (p. 77). Esta observación, hecha por un historiador de la medicina hace alrededor de treinta años, es verdadera sólo hasta cierto punto. En realidad, el interés por estudiar la relación entre las desigualdades sociales y la salud es muy antiguo, y en Europa se remonta más allá del siglo XVIII. El trabajo fundacional de Ramazzini 10 acerca de las relaciones entre el trabajo y la enfermedad, escrito en 1700, constituye un formidable esfuerzo por esclarecer las relaciones entre el lugar ocupado por las personas en la gama de actividades laborales y el desarrollo de patologías específicas. A lo largo de ese siglo se llevaron a cabo otros estudios que relacionaban la pobreza con la mortalidad, la esperanza de vida y las enfermedades que afectaban a la población desfavorecida, lo que culminó con la publicación, en 1790, del tratado La Miseria del Pueblo, Madre de Enfermedades del médico alemán Johann Peter Frank 11.

En 1840, Louis René Villermé publicó un trabajo en Francia comparando la salud de los trabajadores del algodón con la de los propietarios de las fábricas, dando inicio a una larga serie de trabajos similares 12. A lo largo del siglo XIX estos estudios fueron realizados por médicos de gran renombre, como es el caso de RudolfVirchow. Este médico, considerado el padre de la patología, fue el primero en apuntar la necesidad de contar con información certera para identificar de manera científica estas relaciones. En 1848 decía que "las estadísticas médicas serán nuestro patrón de medida: pesaremos cada vida y veremos hacia donde se inclina más la muerte, hacia los trabajadores o hacia los privilegiados" 9 (p. 87). Sin embargo, esta primera generación de estudios sobre la desigualdad social y la enfermedad prácticamente se detuvo al triunfar la teoría infecciosa de la enfermedad, a finales del siglo XIX.

Las investigaciones en el siglo XX retomaron en la década de los cuarenta, como consecuencia del auge del Estado de Bienestar, la escasa efectividad del sistema sanitario para responder a los retos que planteaban los nuevos problemas de salud y el fracaso de las teorías disponibles para explicar la permanencia de las desigualdades sanitarias. Una de las primeras acciones emprendidas fue una extensa investigación que culminó en 1942 con la publicación del Plan Beveridge, que reconoce por primera vez el derecho a la salud y la obligación del Estado de protegerlo 13. Más tarde, al acentuarse la crisis económica mundial iniciada en 1973, los estudios sobre el desarrollo económico y las condiciones de salud crecieron 
exponencialmente. En 1974 se publicó en Canadá el Informe Lalonde, que contenía un interesante marco conceptual sobre los factores sociales que determinan la salud, entre los que destaca la desigualdad socioeconómica; en 1977, la Trigésima Asamblea Mundial de la Organización Mundial de la Salud (OMS) propuso que la reducción del abismo en materia de salud entre las diferentes clases sociales debería ser uno de los objetivos fundamentales de los gobiernos para el año 2000; en 1982 se publicó en Inglaterra el ahora famoso Reporte Black 14 que, entre otras cosas, confirmaba que los estratos socioeconómicos más bajos tenían una mortalidad dos y media veces más alta que los estratos superiores 13. Este reporte, que fue oficial en Gran Bretaña, inició un debate sobre la naturaleza, dimensiones y orígenes de las desigualdades en salud, al reconocer el papel de la estructura socioeconómica en la determinación de la distribución de la salud y el bienestar de la población 13 . El resurgimiento del interés por las desigualdades en salud también se debe a que, a pesar del claro mejoramiento de ciertos indicadores de salud poblacional, las disparidades entre grupos en las condiciones de salud se han ampliado de manera paralela al incremento de las diferencias sociales 15 .

A partir de la publicación del Reporte Black, el desarrollo teórico y empírico de la medición de las desigualdades en salud comenzó una marcha que no se ha detenido. En general, se han usado medidas comparativas surgidas de la epidemiología (diferencias en mortalidad, morbilidad o esperanza de vida), la sociología (diferencias en educación, salario, ocupación o clase social) y la economía (nivel de pobreza, índice de Gini o curva de Lorenz), así como sus posibles combinaciones. Desde el campo de la medicina social se ha propuesto hacer operativa la categoría de clase social, evitando considerar la condición socioeconómica únicamente como un factor más dentro del espectro de elementos que participan en la determinación de la salud 16,17,18,19. En la actualidad, sin embargo, la categoría más utilizada para evaluar estas desigualdades es la de equidad. Esta categoría se sustenta tanto en teorías económicas como en consideraciones de ética y política social, especialmente las desarrolladas a partir de los aportes de John Rawls 20. Algunos de los principales teóricos en este campo han sido Amartya Sen 21, Peter Townsend 14, Margaret Whitehead 22, Michael Marmot 23 y Norman Daniels 24 .

Recientemente la OMS, durante su Asamblea Mundial de 2004, decidió formar una comisión encargada de investigar los determinantes sociales de salud (CSDS-OMS) a fin de realizar estudios $\mathrm{y}$ recomendaciones encaminadas a disminuir las inequidades sanitarias en el mundo. La comisión finalmente fue creada en 2005 y tendrá tres años de existencia. Entre sus objetivos destacan la sistematización de evidencias y de experiencias útiles para formular políticas enfocadas en los determinantes sociales de la salud; el fomento del debate social sobre las acciones que deben emprenderse para enfrentar estos determinantes y la definición de compromisos de medio y largo plazo con vistas a incorporar el tema de las desigualdades en salud en la agenda central de los países y de la propia OMS.

En su sentido moderno, las inequidades en el campo de la salud han sido definidas como "disparidades de salud en un país y entre diferentes países que se consideran improcedentes, injustas, evitables e innecesarias (no irremediables) y que afectan sistemáticamente a poblaciones que han sido hechas vulnerables por las estructuras sociales subyacentes y por las instituciones políticas, económicas y legales" 25 (p. 847). La desigualdad social puede ser resultado de ventajas o desventajas a las que se enfrentan grupos sociales definidos a partir de la condición socioeconómica, el género, la raza-etnia, la religión o la orientación sexual.

En revisiones 26,27 sobre investigaciones empíricas realizadas en América Latina se documentaron 304 informes producidos en la región sobre disparidades socioeconómicas en salud, y se concluyó que: (a) contienen poca información empírica capaz de orientar intervenciones; (b) existen todavía múltiples problemas de diseño, análisis y calidad de la información utilizada, (c) se refieren casi siempre a datos secundarios, y (d) casi nunca se refieren a situaciones locales. Aparentemente, la preocupación de los investigadores ha estado centrada en la comparación de indicadores a escala internacional y siguen usándose indicadores socioeconómicos de origen muy diverso.

\section{Situación de la investigación sobre condiciones socioeconómicas y salud mental}

En los países de altos ingresos ha existido una amplia producción científica sobre la relación entre posición socioeconómica y salud mental, lo cual se refleja en la existencia de varias revisiones 28,29,30,31,32,33,34. Dohrenwend \& Dohrenwend 29 han identificado tres etapas de los estudios realizados dentro del campo de la epidemiología psiquiátrica de los países de altos ingresos. En la primera generación (entre la segunda mitad del siglo XIX y antes del inicio de la segunda guerra mundial) se utilizaron in- 
formantes claves (e.g. médicos) y registros institucionales para identificar los casos de enfermedad psiquiátrica en las comunidades. En la segunda generación (posterior a la segunda guerra mundial) se identificaban los casos mediante entrevistas directas con los residentes de las comunidades realizadas por clínicos o entrevistadores entrenados; en este caso los diagnósticos eran derivados del juicio de los clínicos o mediante escalas de tamizaje. En diversos estudios de estas dos primeras generaciones se reportó de forma consistente una relación negativa entre status socioeconómico y trastornos mentales, incluyendo esquizofrenia, depresión mayor, personalidad antisocial, trastornos por abuso de sustancias y distrés psicológico 29,30.

La tercera generación de estudios de epidemiología psiquiátrica se inició con la publicación del DSM-III en 1980, y se considera que este conjunto de criterios detallados y explícitos permitió mayor precisión en los diagnósticos psiquiátricos. Aunado a ello se desarrollaron métodos para mejorar la validez y confiabilidad de los diagnósticos (e.g. procedimientos en los que primero se aplican escalas de tamizaje y posteriormente se confirman los diagnósticos con entrevistas psiquiátricas estandarizadas) 29 . En varios estudios de esta generación también se observó la relación negativa entre status socioeconómico y trastornos mentales 30 .

Respecto a la producción científica en América Latina, Almeida-Filho 35 planteó que existían dos hipótesis que podrían explicar la distribución de los trastornos mentales: (a) la hipótesis sociocultural, según la cual los trastornos eran debidos al estrés generado por los rápidos cambios culturales asociados con la migración del campo a las ciudades (aculturación), ésta última generada por el proceso de modernización y (b) la hipótesis de la exclusión económica, en la que los trastornos son resultado del desempleo, el cual es una expresión de la exclusión de los procesos productivos modernos, siendo la exclusión (i.e. la existencia de un ejército industrial de reserva) una característica estructural del sistema económico capitalista periférico. Para abordar esta confrontación entre las dos hipótesis, el autor identificó tres aproximaciones: (i) Los estudios "pre-epidemiológicos" que abarcan reportes de casos clínicos de personas referidas a servicios de psiquiatría o estudios con pequeñas muestras $(\mathrm{n}<270)$ de sujetos residentes de áreas marginadas en los que se reportó que diferentes indicadores de migración y de situación socioeconómica (e.g. ingreso y desempleo) se relacionaron con mayor distrés psicológico; (ii) Las encuestas comunitarias (realizadas entre 1950 y 1970) que se caracterizan por la aplicación de escalas psico- métricas sin validación en muestras representativas de pequeñas áreas geográficas o comunidades en las que se continuó estudiando la relación de la migración con el distrés psicológico; (iii) Los estudios epidemiológicos (realizados desde 1970) en los que se utilizó muestreo probabilístico y se identificaban los casos mediante instrumentos de tamizaje y posteriormente se confirmaban los diagnósticos mediante entrevistas psiquiátricas. En varias investigaciones se reiteraron los hallazgos sobre el mayor riesgo de distrés psicológico en emigrantes, aunque también se evidenció que dicha asociación desaparecía o se atenuaba cuando se consideraban aspectos socioeconómicos.

Recientemente, Patel et al. 36 compararon investigaciones realizadas en cuatro países en desarrollo (incluyendo las ciudades de Santiago, en Chile, Olinda y Pelotas, en Brasil) sobre la relación del sexo y la pobreza con los trastornos mentales comunes (término que hace referencia a síntomas somáticos, de ansiedad y depresión). Patel \& Kleiman 37 publicaron una revisión de estudios realizados en países de ingreso bajo y medio sobre la relación entre pobreza (medida a través de indicadores de clase social o status socioeconómico) y trastornos mentales comunes. Los autores identificaron once estudios de seis países, de los cuales tres son de Brasil y uno de Chile, y los siete restantes de África y Asia.

El objetivo de este trabajo es presentar una actualización de la revisión de la literatura sobre la relación entre la condición socioeconómica y trastornos mentales para incluir los estudios publicados después de la revisión de AlmeidaFilho 35 y aquellos que no fueron considerados en revisiones previas 37 por estar escritos en español o portugués. Hay que añadir que las revisiones anteriores no han considerado el uso, abuso y dependencia a alcohol y otras sustancias.

\section{Material y métodos}

La revisión se restringió a los trabajos publicados a partir de 1982. La búsqueda biblio-hemerográfica sobre investigaciones que hayan analizado la relación entre estratificación socioeconómica y trastornos mentales en población latinoamericana se realizó consultando las siguientes bases de datos: MEDLINE (http://www.ncbi.nlm.nih. gov/entrez/), Scientific Electronic Library Online (SciELO; http:/ /www.scielo.org), base de datos referencial producida por el Centro de Documentación e Información en Psiquiatría y Salud Mental (CDISPM) del Instituto Nacional de Psiquiatría, enlaces a revistas electrónicas con acceso gratuito en Internet a través del CDISPM, Periódica y Clase de la Dirección General de Bibliotecas de 
la Universidad Nacional Autónoma de México (UNAM), Índice de Revistas Médicas Latinoamericanas (IMBIOMED) y las compilaciones incluidas en las EBSCO HOST Research Databases (Academic Search Premier, Fuente Académica, Medic Latina, Medline, Psychology and Behavioral Sciences y Sociological Collection).

En la segunda etapa del estudio, los trabajos identificados fueron organizados de la siguiente forma: los que abordan la relación de la estratificación con indicadores de distrés psicológico o trastornos mentales en adultos (Tabla 1); los que describen las características de pacientes hospitalizados por conducta suicida, los que se enfocan en uso y abuso de sustancias en adultos (Tabla 2); aquellos que analizan la asociación con indicadores de salud mental en adolescentes y jóvenes (Tabla 3); y los que evalúan el uso y consumo de sustancias en adolescentes y jóvenes (Tabla 4). Para elaborar las tablas se resumieron las características y los principales resultados de los estudios, esta información puede ser solicitada a los autores.

\section{Resultados}

En la mayoría de los estudios (Tabla 1) se ha observado que en los adultos de países de América Latina el pertenecer a estratos socioeconómicos bajos se asocia con mayor riesgo de: intento de suicidio 38,39 , sintomatología depresiva evaluada mediante escalas psicométricas $40,41,42,43,44$, trastornos mentales comunes (básicamente depresión y ansiedad) evaluados a través de cuestionarios validados contra entrevistas psiquiátricas estandarizadas $45,46,47,48,49,50,51,52,53,54,55,56,57,58,59$ y con trastornos mentales evaluados mediante entrevistas diagnósticas (e.g. CIDI) que permiten diferenciar cuadros clínicos específicos como trastornos del estado de ánimo, fobias $60,61,62,63,64,65,66,67$, ansiedad 68,69, disfunción sexual y déficit cognitivo 70. En un estudio ecológico 71 existió asociación positiva entre la tasa de mortalidad por suicidio y el porcentaje de población pobre. Sin embargo, en algunos estudios la relación tuvo forma de "U" (escolaridad y trastorno de pánico 70) o de "U" invertida (trastornos mentales y un índice de nivel socioeconómico ${ }^{72}$ ). En un estudio chileno 68,69 se encontraron tanto asociaciones positivas como negativas según la medida de morbilidad utilizada (prevalencia en la vida, en los últimos seis meses o en el último mes). En otras investigaciones no existió asociación (escolaridad y ocupación con el intento de suicidio 73,74 , intento de suicidio e ingreso 75 , suicidio consumado y un indicador socioeconómico de área geográfica 76).
En estudios en adultos en los que se ha utilizado un solo indicador socioeconómico se ha reportado que la escolaridad se relaciona negativamente con trastornos en general $68,69,70$ o con sintomatología depresiva 42 - aunque las diferencias no fueron significativas -, pese a que en otros estudios no se ha observado asociación con trastornos mentales 46. En una investigación se reportó que el desempleo aumentó el riesgo de sintomatología depresiva 67 . En otros estudios no existió relación entre los trastornos mentales y un índice compuesto por escolaridad, actividad laboral y vivienda 72 , mientras que otra investigación en la que se utilizó un indicador compuesto de estrato socioeconómico sí se observó relación con sintomatología psiquiátrica 45 . El ingreso no se relacionó con el intento de suicidio ${ }^{75}$, pero sí con sintomatología depresiva ${ }^{41}$. Un indicador de condiciones de vida basado en áreas geográficas no se asoció con el suicido consumado 76 . Considerando las investigaciones en las que se ha evaluado más de un indicador, en una experiencia se observó que la escolaridad, pero no el ingreso, se relacionó con trastornos mentales 62,63. La depresión se relacionó con la escolaridad, pero no con un índice que incorporaba la clase ocupacional 57. En otro estudio, en modelos de regresión logística múltiple se observó que la escolaridad, la disminución reciente del ingreso y la calidad de la vivienda se relacionaron con los trastornos mentales comunes, mientras que la relación desapareció con el ingreso, el hacinamiento y la propiedad de la vivienda 53,54,55. En una investigación, el desempleo, la baja escolaridad y la residencia en un sub-distrito con condiciones socioeconómicas deficientes fueron factores de riesgo para trastornos mentales comunes; sin embargo, no se observó dicha asociación con el ingreso 50. En otro estudio, la depresión se relacionó con la situación laboral, pero no con la escolaridad y el ingreso familiar, aunque los tres indicadores se asociaron con la fobia social 65,66. Los trastornos del estado de ánimo se han asociado con la baja escolaridad, pero no con los ingresos 64 . En una experiencia, el bajo ingreso - pero no la escolaridad - se relacionó con trastornos mentales comunes 59 . Al analizar la relación de trastornos mentales con escolaridad, ingreso, tipo de ocupación, analfabetismo, condiciones de vivienda y posesión de bienes, sólo se observó relación con los dos primeros 51,52. En otros estudios tanto la baja escolaridad como el desempleo se relacionaron con mayor prevalencia de intento de suicidio 40 y síntomas depresivos 44 . El desempleo y bajos niveles de ingreso y escolaridad se relacionaron con mayor riesgo de trastornos psiquiátricos menores 49 . El desempleo, pero no la escolaridad, se 
Tabla 1

Resumen de estudios que relacionan la condición socioeconómica con trastornos mentales en adultos de América Latina.

\begin{tabular}{|c|c|c|c|c|c|c|}
\hline $\begin{array}{l}\text { Indicador } \\
\text { socioeconómico }\end{array}$ & TMG/TMC * & $\begin{array}{l}\text { Conducta } \\
\text { suicida ** }\end{array}$ & $\begin{array}{c}\text { Distrés psicológico/ } \\
\text { Sintomatología } \\
\text { de depresión } \star \star \star\end{array}$ & Afecto \# & Ansiedad \#\# & $\begin{array}{l}\text { Otros } \\
\text { trastornos } \\
\text { mentales }\end{array}$ \\
\hline \multicolumn{7}{|l|}{ Escolaridad } \\
\hline Relación no-lineal & Penayo et al. 46 & & & & Canino et al. 70 & \\
\hline Relación negativa & $\begin{array}{l}\text { Lima et al. 49; Blue 50; } \\
\text { Ludermir \& Lewis 51; } \\
\text { Araya et al. 53; } \\
\text { Vicente et al. } 68 ; \\
\text { Canino et al. } 70\end{array}$ & & $\begin{array}{l}\text { Villanueva et al. 42; } \\
\text { Illanes et al. } 43 ; \\
\text { Gazalle et al. }{ }^{44 ;} \\
\text { Almeida-Filho } \\
\text { et al. } 57 \text {; } \\
\text { Maia et al. } 58\end{array}$ & $\begin{array}{l}\text { Caraveo-Anduaga } \\
\text { et al. 62; } \\
\text { Andrade et al. } 64\end{array}$ & $\begin{array}{l}\text { Caraveo-Anduaga } \\
\text { et al. 62; } \\
\text { Vorcaro et al. } 65 ; \\
\text { Vicente et al. } 68\end{array}$ & Canino et al. 70 \\
\hline Sin relación & & $\begin{array}{l}\text { Borges et al. 38; } \\
\text { Mondragón } \\
\text { et al. } 73,74 ; \\
\text { Gómez-Restrepo } \\
\text { et al. } 75\end{array}$ & & & & \\
\hline \multicolumn{7}{|l|}{ Ingreso } \\
\hline Relación positiva & & Botega et al. 39 & & & & Vicente et al. 68 \\
\hline Relación no-lineal & & & & & $\begin{array}{l}\text { Caraveo-Anduaga } \\
\text { et al. } 62\end{array}$ & \\
\hline Relación negativa & $\begin{array}{l}\text { Lima et al. 49; Blue 50; } \\
\text { Ludermir \& Lewis 51; } \\
\text { Araya et al. 53; } \\
\text { Kac et al. 59; } \\
\text { Berenzon et al. 60; } \\
\text { Vicente et al. } 68\end{array}$ & & $\begin{array}{l}\text { Jadresic \& Araya 41; } \\
\text { Maia et al. } 58\end{array}$ & Vicente et al. 68 & $\begin{array}{l}\text { Vorcaro et al. } 65 ; \\
\text { Vicente et al. } 68\end{array}$ & \\
\hline Sin relación & & & & Andrade et al. 64 & Andrade et al. 64 & \\
\hline \multicolumn{7}{|l|}{ Desempleo } \\
\hline Asociación & $\begin{array}{l}\text { Lima et al. 49; } \\
\text { Araya et al. 53; } \\
\text { Almeida-Filho } \\
\text { et al. } 56\end{array}$ & $\begin{array}{l}\text { Borges et al. }{ }^{38} \text {; } \\
\text { Medina-Mora } \\
\text { et al. } 40\end{array}$ & $\begin{array}{l}\text { Medina-Mora } \\
\text { et al. } 40 \text {; Gazalle } \\
\text { et al. } 44\end{array}$ & $\begin{array}{l}\text { Vorcaro et al. 65; } \\
\text { Gómez-Restrepo } \\
\text { et al. } 75\end{array}$ & Vorcaro et al. 65 & \\
\hline Sin asociación & & $\begin{array}{l}\text { Mondragón } \\
\text { et al. } 73,74\end{array}$ & & & & \\
\hline \multicolumn{7}{|l|}{ Clase ocupacional } \\
\hline Relación positiva & $\begin{array}{l}\text { Blue 50; } \\
\text { Ludermir \& Lewis 51; } \\
\text { Araya et al. } 53\end{array}$ & & & $\begin{array}{l}\text { Almeida-Filho } \\
\text { et al. } 57\end{array}$ & & \\
\hline \multicolumn{7}{|l|}{$\begin{array}{l}\text { Vivienda, posesión } \\
\text { de bienes; residencia, } \\
\text { índices compuestos } \\
\text { o no descritos }\end{array}$} \\
\hline Relación positiva & $\begin{array}{l}\text { Blue 50; Araya } \\
\text { et al. } 53\end{array}$ & & & & & \\
\hline Relación no-lineal & Vicente et al. 72 & & & & & \\
\hline Relación negativa & Di Marco 45 & & Maia et al. 58 & Berenzon et al. 60 & Berenzon et al. 60 & \\
\hline Sin relación & & Rivera 76 & & & & \\
\hline
\end{tabular}

* Trastornos comunes o menores diagnosticados con escalas de tamizaje o algún trastorno diagnosticado con entrevista psiquiátrica;

** Ideación o intento de suicidio;

*** Evaluados mediante escalas psicométricas;

\# Incluye depresión, distimia diagnosticados con entrevista psiquiátrica;

\#\# Incluye fobias, agorafobia diagnosticadas con entrevista psiquiátrica. 
relacionó con el intento de suicidio 38 ; o bien ni la escolaridad, ni el desempleo se relacionaron con la ideación de suicidio 73,74. También se ha reportado que los síntomas depresivos se relacionan con baja escolaridad y la desocupación, pero no el ingreso 43. Los grupos de menor ingreso, que habitaban en sectores marginados y con menos escolaridad tuvieron mayor riesgo de síntomas psiquiátricos, sin embargo, las diferencias de acuerdo a la última variable no fueron estadísticamente significativas 58 . La situación laboral y el ingreso se relacionaron con la idea suicidio, pero no la escolaridad 39.

En los estudios en los que se han analizado las características de pacientes hospitalizados por intento de suicidio, en algunos se ha observado que existe predominio de población de baja escolaridad $77,78,79,80,81$, desempleados 78,82 , personas en ocupaciones de bajo status $77,83 \mathrm{O}$ estudiantes y amas de casa $79,83,84,85,86$, aunque en otros reportes se ha observado predominio de sujetos con alta escolaridad 82 .

Respecto al uso y abuso de sustancias (Tabla 2), aunque en México la probabilidad de que en los hogares se compre tabaco se incrementa con el ingreso 87,88, en cuatro estudios con adultos se reportó que el nivel socioeconómico se relaciona negativamente con el tabaquismo 89,90,91,92,93. En la población adulta de América Latina el consumo de alcohol se incrementa conforme aumenta el estrato socioeconómico 92,94,95,96. En el caso de la dependencia al alcohol (alcoholismo) o los problemas derivados de su consumo se ha observado mayor riesgo en las personas de menor nivel socioeconómico 64,90,91,95,97,98,99,100,101. Aunque en otros estudios se observa el mismo patrón, las diferencias desaparecen al ajustar por otras variables 94 y en otras experiencias la relación tuvo forma de "U" 70 o es positiva 68,69,72,94. En grupos de auto-ayuda o centros de atención de alcoholismo se ha observado predominio de personas de baja escolaridad 102,103,104. Sólo se identificó un estudio en el que se haya evaluado la tasa de recuperación de alcoholismo y dependencia a otras drogas, en el cual no se encontraron diferencias por nivel socioeconómico 105.

Con relación a las drogas ilegales, en un estudio en Chile 92 no se observó un patrón claro de consumo de cocaína y marihuana en función del nivel socioeconómico. Sin embargo, datos de México 106 sugieren que en los últimos años el aumento en el consumo de cocaína se ha dado sobretodo en los estratos bajos; en la población de Argentina el uso frecuente de marihuana, cocaína y estimulantes fue más prevalente en los estratos bajos 100, de igual modo, en México el uso de drogas ilegales (marihuana, cocaína, inhalables, alucinógenos y heroína) fue más co- mún entre las personas con menor escolaridad 107. Con relación a las drogas médicas, entre los grupos con mejor condición socioeconómica es más frecuente el uso de anorexigénicos 49 y sedantes 100 , mientras que el uso de ansiolíticos no varía en función de la condición socioeconómica 92 .

En estudiantes de enseñanza media la ideación suicida se relacionó negativamente con la escolaridad del jefe de familia 108 (Tabla 3). En otro grupo de adolescentes, el efecto del estrés psicosocial sobre síntomas depresivos fue más evidente entre aquellos de estratos bajos 109. En adolescentes de Chile el nivel socioeconómico se correlacionó negativamente con la ansiedad, pero positivamente con la autoestima 110 y en estudiantes universitarios de Colombia existió relación inversa entre estrato socioeconómico y depresión 111. Los trastornos de la conducta son más frecuentes en niños y adolescentes de estratos bajos 112,113.

En adolescentes (Tabla 4) el consumo de alcohol se asocia positivamente con el nivel socioeconómico 110,114,115,116,117,118,119,120,121,122,123, 124,125,126, al tiempo que la embriaguez tiene una distribución contraria 115. Aunque en un estudio se observó que la distribución del consumo de alcohol 127 en función del nivel socioeconómico tenía una forma de "U", es decir, su consumo es más frecuente en los extremos de la jerarquía social.

Aunque en la mayoría de los estudios se ha reportado que el consumo de tabaco es más frecuente en los adolescentes de estratos altos 116,119, 120,121,122,126,128, en otros se reportó la tendencia contraria 129; además, en un caso 127 , el nivel socioeconómico no se relacionó con el consumo de tabaco.

En el caso de las drogas ilegales, en un estudio en México el consumo de marihuana fue más frecuente en el estrato medio-alto 128; en Brasil el consumo de drogas psicoactivas ilegales (medicamentos, marihuana, cocaína, alucinógenos y opiáceos) también fue más frecuente en las clases superiores 127; en estudiantes de Morelos, México, la prevalencia del consumo de drogas (marihuana, cocaína, heroína, pegamentos inhalantes y solventes) fue más elevada en el nivel alto 126; en estudiantes de escuelas de Brasil se observó la misma tendencia con el consumo de marihuana 122 y de uso pesado de drogas 124,125. Respecto a colegios públicos de Costa Rica, en los privados fue más frecuente el uso de tranquilizantes, aunque lo contrario se observó con el uso de drogas ilícitas 120. Por el contrario, en Colombia en el estrato bajo fue más frecuente el consumo de marihuana y cocaína 116; al igual que en un estudio en Guadalajara, México, don- 
Resumen de estudios que relacionan la condición socioeconómica con trastornos por uso de sustancias en adultos de América Latina.

\begin{tabular}{|c|c|c|c|c|c|}
\hline $\begin{array}{l}\text { Indicador } \\
\text { socioeconómico }\end{array}$ & $\begin{array}{l}\text { Consumo } \\
\text { de alcohol }\end{array}$ & $\begin{array}{c}\text { Dependencia y abuso } \\
\text { en el consumo de alcohol }\end{array}$ & Tabaquismo & $\begin{array}{l}\text { Drogas } \\
\text { ilegales }\end{array}$ & $\begin{array}{l}\text { Drogas } \\
\text { médicas }\end{array}$ \\
\hline \multicolumn{6}{|l|}{ Escolaridad } \\
\hline Relación positiva & $\begin{array}{l}\text { Almeida \& } \\
\text { Coutinho } 94 ; \\
\text { Bejarano \& Carvajal } 99\end{array}$ & & & & Lima et al. 49 \\
\hline Relación no-lineal & & Canino et al. 70 & & & \\
\hline Relación negativa & & $\begin{array}{l}\text { Moreira et al. 90; } \\
\text { Villatoro-Velásquez et al. 97; } \\
\text { Medina et al. 98; } \\
\text { Bejarano \& Carvajal 99; } \\
\text { Rosovsky et al. 102; } \\
\text { Bejarano \& Carvajal 103; } \\
\text { Benassini \& Guzmán } 104\end{array}$ & $\begin{array}{l}\text { Vásquez-Segovia } \\
\text { et al. } 87 ; \\
\text { Lolio et al. } 89 ; \\
\text { Moreira et al. } 90 ; \\
\text { Kroeff et al. } 93\end{array}$ & Cravioto et al. 107 & \\
\hline Sin relación & & $\begin{array}{l}\text { Almeida \& Coutinho } 94 ; \\
\text { Almeida-Filho et al. } 96\end{array}$ & & & \\
\hline \multicolumn{6}{|l|}{ Ingreso } \\
\hline $\begin{array}{l}\text { Relación positiva } \\
\text { Relación no-lineal }\end{array}$ & Almeida \& Coutinho 94 & Vicente et al. 68 & \multicolumn{2}{|l|}{ Vásquez-Segovia et al. 87} & Lima et al. 49 \\
\hline Relación negativa & & $\begin{array}{l}\text { Moreira et al. }{ }^{90} \\
\text { Medina et al. } 98\end{array}$ & \multicolumn{2}{|l|}{$\begin{array}{l}\text { Sesma-Vásquez et al. } 88 \text {; } \\
\text { Lolio et al. } 89 ; \\
\text { Moreira et al. } 90\end{array}$} & \\
\hline Sin relación & & $\begin{array}{l}\text { Andrade et al. 64; } \\
\text { Almeida \& Coutinho } 94\end{array}$ & & & \\
\hline \multicolumn{6}{|l|}{ Desempleo } \\
\hline Asociación & & $\begin{array}{l}\text { Andrade et al. 64; } \\
\text { Bejarano \& Carvajal 103; } \\
\text { Benassini \& Guzmán } 104\end{array}$ & & & \\
\hline \multicolumn{6}{|l|}{ Sin asociación } \\
\hline \multicolumn{6}{|l|}{ Clase ocupacional } \\
\hline Relación positiva & Fuentealba et al. 92 & $\begin{array}{l}\text { Vicente et al. }{ }^{72} \text {; } \\
\text { Almeida-Filho et al. } 96\end{array}$ & Moreira et al. 90 & & Míguez 100 \\
\hline \multicolumn{6}{|l|}{ Relación no-lineal } \\
\hline Relación negativa & & $\begin{array}{l}\text { Andrade et al. 64; } \\
\text { Medina et al. 98; } \\
\text { Míguez 100; } \\
\text { Mendoza-Sassi \& Béria } 101\end{array}$ & Fuentealba et al. 92 & $\begin{array}{l}\text { Míguez 100; } \\
\text { Unikel et al. } 106\end{array}$ & \\
\hline Sin relación & & & & Fuentealba et al. 92 & \\
\hline
\end{tabular}

de el consumo de drogas (marihuana, heroína, cocaína, morfina, crack, hachís y anfetaminas) fue más frecuente en el estrato inferior 121; en un estudio en Brasil las clases bajas tuvieron mayor riesgo de consumo de cocaína, aunque las diferencias fueron marginales 123 .

El consumo de anfetaminas 128 y tranquilizantes 119,128 ha sido más frecuente en adolescentes de estratos altos. En estudios en los que se ha indagado el consumo de drogas en general (alcohol, tabaco y sustancias ilegales), se ha observado que éste es más elevado en los estratos altos 124,125,130. En tres estudios 119,128,131 se reportó que el uso de inhalantes es más frecuente en sujetos de estratos bajos. En una investigación se encontró que el consumo de marihuana, anfetaminas y sedantes 119 de acuerdo al nivel socioeconómico tenía una forma de "U". También se ha encontrado que el nivel socioeconómico no se relacionó con el consumo de drogas (sin incluir alcohol y tabaco) 119,132 o con el uso de marihuana 110. En otra investigación no se observó una relación lineal entre posición socioeconómica y uso de alcohol y marihuana 128. 
Resumen de estudios que relacionan la condición socioeconómica con trastornos mentales en adolescentes de América Latina.

\begin{tabular}{|c|c|c|c|}
\hline $\begin{array}{l}\text { Indicador } \\
\text { socioeconómico }\end{array}$ & $\begin{array}{l}\text { Ideación } \\
\text { suicida }\end{array}$ & $\begin{array}{l}\text { Distrés psicológico/ } \\
\text { Sintomatología de depresión * }\end{array}$ & $\begin{array}{c}\text { Trastornos de la } \\
\text { conducta }\end{array}$ \\
\hline \multicolumn{4}{|l|}{ Escolaridad } \\
\hline Relación negativa & López et al. 108 & & \\
\hline \multicolumn{4}{|c|}{ Residencia o tipo de escuela } \\
\hline \multirow[t]{3}{*}{ Relación negativa } & & González-Forteza et al. 109; & \\
\hline & & McWhriter et al. 110; & \\
\hline & & Miranda et al. 111 & \\
\hline \multicolumn{4}{|c|}{ Índices compuestos o no descritos } \\
\hline \multirow[t]{2}{*}{ Asociación negativa } & & & Bralio et al. 112; \\
\hline & & & Pineda et al. 113 \\
\hline
\end{tabular}

* Evaluados mediante escalas psicométricas.

\section{Discusión}

En la mayoría de los estudios de Latinoamérica con adultos se ha observado que la posición socioeconómica se relaciona negativamente con indicadores de trastornos mentales y, aunque existen menos estudios, se ha observado la misma tendencia en el caso de adolescentes y jóvenes; lo anterior coincide con los resultados de investigaciones realizadas en países de altos ingresos 29,30,31,32,34,133,134. Las investigaciones con adultos de América Latina en las que no se observó asociación el evento estudiado fue el intento de suicidio o el suicidio consumado $73,74,75,76$; en otras investigaciones sobre el mismo evento las relaciones han sido negativas 38,39 .

El hallazgo general de la relación negativa entre estratificación y salud mental en adultos puede ser explorado en detalle al considerar las discrepancias que se observan en función del indicador de posición socioeconómica que se utilice. Por ejemplo, con datos de la National Comorbility Survey (NCS) se observó que mientras la escolaridad se relacionaba negativamente con la prevalencia en el último mes de ataques de pánico, trastorno de pánico y pánico con agarofobia, en el caso del ingreso dichas asociaciones no se observaron; por otro lado, los trastornos de la personalidad antisocial se relacionaron tanto con la escolaridad como con el ingreso 31 . En una revisión 32 sobre encuestas desarrolladas en países industrializados se identificó que el indicador de la posición social que se asociaba en menos estudios fue la clase social ocupacional, mientras que los indicadores que más se relacionaron fueron la escolaridad, el desempleo y el ingreso. En el caso de América Latina, la escolaridad se ha asociado con síntomas de depresión 43,57, intento de suicido 40,44 y trastornos mentales del estado de ánimo y ansiedad 62,63,64 y trastornos en general 49,50,51,52,53,54,55,68,69,70; otros tres estudios, aunque observaron la misma tendencia, no encontraron diferencias significativas 42,58,59; en dos estudios más dicho indicador no se relacionó con síntomas de depresión 46,65,66, y en otras experiencias tampoco se vinculó la escolaridad con ideación e intento de suicido $38,39,73,74$. El desempleo por su parte, se ha relacionado con mayor riesgo de síntomas depresivos $43,44,67$, trastornos mentales comunes 49,50 e intento de suicidio 38,40 , aunque en dos estudios dicha variable no aumentó el riesgo de ideación suicida 73,74.

En la mayoría de los estudios la escolaridad se relaciona con sintomatología indicativa de trastornos mentales, lo cual puede atribuirse a que dicho indicador refleja adecuadamente la estratificación socioeconómica al definir las posibilidades de inserción ocupacional e ingreso, y a diferencia de estas dos últimas variables tiene la ventaja de que las personas la pueden reportar con mayor precisión 135 . En el caso de la conducta suicida, sólo en uno de cuatro estudios se observó asociación con la escolaridad; mientras que el intento de suicidio, pero no la ideación de suicidio, se relaciona con mayor consistencia con el desempleo. Esto puede deberse a que dicho evento se presenta ante en situaciones críticas o agudas 55 , mientras que la escolaridad es una variable que mide un atributo permanente o estable.

Algunos estudios reportan que el ingreso no se relaciona con indicadores de salud mental 43 , $50,53,54,55,62,63,64,65,66$, aunque en otros sí se observa 
Tabla 4

Resumen de estudios que relacionan la condición socioeconómica con trastornos por uso de sustancias en adolescentes de América Latina.

\begin{tabular}{|c|c|c|c|c|c|c|c|}
\hline $\begin{array}{l}\text { Indicador } \\
\text { socioeconómico }\end{array}$ & $\begin{array}{l}\text { Consumo } \\
\text { de alcohol }\end{array}$ & $\begin{array}{l}\text { Dependencia } \\
\text { y abuso en el } \\
\text { consumo de } \\
\text { alcohol }\end{array}$ & Tabaquismo & $\begin{array}{l}\text { Drogas } \\
\text { ilegales }\end{array}$ & $\begin{array}{l}\text { Drogas } \\
\text { médicas }\end{array}$ & Inhalables & $\begin{array}{c}\text { Sustancias } \\
\text { en } \\
\text { general * }\end{array}$ \\
\hline \multicolumn{8}{|l|}{ Escolaridad } \\
\hline Relación positiva & $\begin{array}{l}\text { Oderich et al. 118; } \\
\text { Medina-Mora } \\
\text { et al. } 119\end{array}$ & & $\begin{array}{l}\text { Medina-Mora } \\
\text { et al. } 119\end{array}$ & & & & $\begin{array}{l}\text { Medina-Mora } \\
\text { et al. } 119\end{array}$ \\
\hline Relación no-lineal & & & & & & $\begin{array}{l}\text { Medina-Mora } \\
\text { et al. } 119\end{array}$ & \\
\hline Relación negativa & & & & & & $\begin{array}{l}\text { Medina-Mora } \\
\text { et al. 119; } \\
\text { Berenzon et al. } 131\end{array}$ & \\
\hline Sin relación & & & & & & & $\begin{array}{l}\text { Medina-Mora \& } \\
\text { Rojas } 132\end{array}$ \\
\hline \multicolumn{8}{|l|}{ Ingreso } \\
\hline Relación positiva & $\begin{array}{l}\text { Badilla \& } \\
\text { Barquero } 117\end{array}$ & & & & & & \\
\hline \multicolumn{8}{|l|}{ Clase ocupacional } \\
\hline Relación positiva & $\begin{array}{l}\text { Queiroz et al. } 122 ; \\
\text { Baus et al. } 123\end{array}$ & Soldera et al. 124 & Queiroz et al. 122 & Queiroz et al. 122 & & & $\begin{array}{l}\text { Caballero et al. }{ }^{121 ;} \\
\text { Muza et al. } 127 ; \\
\text { Souza \& Martins } 130\end{array}$ \\
\hline $\begin{array}{l}\text { Sin relación } \\
\text { Relación negativa }\end{array}$ & & & Muza et al. 127 & Baus et al. 123 & & & \\
\hline \multicolumn{8}{|l|}{ Residencia o tipo } \\
\hline Relación positiva & $\begin{array}{l}\text { Maya \& García 114; } \\
\text { Caroma et al. 115; } \\
\text { Bergonzoli et al. } 116 ; \\
\text { Sandí \& Díaz } 120 ; \\
\text { Caballero et al. } 121\end{array}$ & & $\begin{array}{l}\text { Bergonzoli et al. } 116 \\
\text { Sandí \& Díaz 120; } \\
\text { Caballero et al. } 121 ; \\
\text { Castro et al. } 128\end{array}$ & 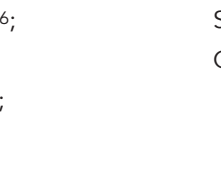 & $\begin{array}{l}\text { Sandí \& Díaz 120; } \\
\text { Castro et al. } 128\end{array}$ & & \\
\hline Relación no-lineal & & Castro et al. 128 & & Castro et al. 128 & & & \\
\hline Relación negativa & & Caroma et al. 115 & $\begin{array}{l}\text { Velásquez } \\
\text { et al. } 129\end{array}$ & $\begin{array}{l}\text { Bergonzoli et al. }{ }^{116} \\
\text { Sandí \& Díaz } 120\end{array}$ & & Castro et al. 128 & Caballero et al. 121 \\
\hline $\begin{array}{l}\text { Índices compuestos } \\
\text { o no descritos }\end{array}$ & \multicolumn{6}{|c|}{ Índices compuestos } & \\
\hline $\begin{array}{l}\text { Asociación } \\
\text { positiva }\end{array}$ & McWhriter et al. 110 & & & & & & $\begin{array}{l}\text { Herrera-Vásquez } \\
\text { et al. } 126\end{array}$ \\
\hline Sin asociación & & & & McWhriter et al. 110 & & & \\
\hline
\end{tabular}

* Incluye grupos de drogas legales, ilegales y/o alcohol.

tal asociación 39,41,49,51,52,58,68,69. Es probable que la falta de asociación con el ingreso se deba a que ésta es una variable difícil de evaluar debido a las exigencias de cálculo y de memoria que implica para los entrevistados, así como a los sesgos en su reporte por ser un tema sensible.

Existen menos estudios en los que se hayan evaluado índices socioeconómicos basados en áreas geográficas y en características de la vivienda; los primeros se han relacionado con trastornos mentales comunes 50 o con síntomas psiquiátricos 58; sin embargo, no se han asociado con el suicidio consumado 76. Las características de la vivienda se relacionaron con trastornos comunes 53,54,55, aunque en otras investigaciones no se observó tal asociación 51,52. 
Respecto a la medición de la salud mental o trastornos mentales, aunque es común que en las investigaciones se hayan utilizado escalas de distrés psicológico 40,41,42,43,44,45,49,50,53,57,58,73,74, cada vez es más frecuente que se recurra a instrumentos de tamizaje para después confirmar diagnósticos mediante entrevista psiquiátrica $46,47,51,59$ o a entrevistas psiquiátricas estandarizas que utilizan clasificaciones aceptadas internacionalmente (e.g. DSM o CIE) 60,62,64,65,67,68,69, 70,72 . El uso de los dos últimos procedimientos, además de aumentar la posibilidad de comparación entre estudios, reduce sesgos producidos por el uso de escalas psicométricas, ya que los individuos de los estratos socioeconómicos bajos tienden a sub-reportar sintomatología psiquiátrica 136,137,138 y los auto-reportes pueden ser afectados por estados de afectividad negativa 139 .

Las investigaciones en las que se caracteriza a personas que intentaron suicidarse o se suicidaron tienen las limitaciones de la epidemiología descriptiva 139. Estos estudios son afectados por sesgos de selección, ya que reflejan los rasgos de las personas que tienen acceso a los servicios, lo que depende del tipo de población que atienden los hospitales. Así, si los pacientes derivan de hospitales privados los suicidas son tipificados de estratos altos; mientras que si se describe a las personas que acuden a hospitales públicos, éstas serán principalmente de baja condición socioeconómica.

La mayor parte de la evidencia producida en América Latina indica que tanto en adolescentes $110,114,115,116,117,118,119,120,121,122,123,124,125,126$ como en adultos 92,94,95,96 el consumo de alcohol es mayor entre las personas con mejores condiciones; mientras que con el abuso y dependencia al alcohol la tendencia es contraria (adultos 95,97,98,90,91, 99,100,101,102,103,104; adolescentes 115). La evidencia para las últimas condiciones es menos contundente, pues existen estudios en los que no se ha observado tal relación 68,69,70,72,94,96. En Estados Unidos de Norteamérica también la escolaridad y el ingreso se relacionaron negativamente con el abuso y dependencia al alcohol ${ }^{133}$. El costo del alcohol, las normas socioculturales y el nivel de estrés pueden ser factores que modifiquen la relación del nivel socioeconómico con el consumo, el abuso y la dependencia al alcohol. Es posible que el consumo de alcohol sea más frecuente entre los estratos altos, debido a que es una norma sociocultural dentro de esos grupos, además de que cuentan con los recursos para adquirirlo; mientras que el abuso y la dependencia al alcohol son más frecuentes entre los estratos bajos, debido a que se enfrentan con mayor frecuencia a diferentes estresores ${ }^{99}$. Sólo se identificó un estudio sobre la recuperación de dependencia al alcohol y otras drogas 105 en el que no se observaron diferencias socioeconómicas; sin embargo, puede existir sesgo de selección pues las personas de estratos altos tienden a acudir con más frecuencia a los servicios de salud.

Con algunas excepciones 127,129, en los adolescentes el tabaquismo es más frecuente entre los que provienen de hogares con mejores condiciones socioeconómicas 116,119,120,121,122,126,128; sin embargo, entre los adultos la mayor probabilidad de tabaquismo la tienen los de peor situación económica 89,90,91,92,93. Esta discrepancia puede ser resultado de que los adolescentes de estratos altos al tener mayor capacidad adquisitiva comienzan a adquirir tabaco más tempranamente, pero al llegar a la vida adulta las personas de esos estratos buscan con mayor frecuencia abandonar ese hábito ${ }^{33}$, al tiempo que cada vez más adultos de los estratos bajos iniciarán o continuarán consumiendo tabaco.

$\mathrm{Al}$ igual que en adultos de países de altos ingresos 33,133, en los de América Latina el consumo de drogas ilegales en general 107, cocaína 100,106 y marihuana 100 es más frecuente entre las personas de nivel socioeconómico inferior, aunque existen experiencias en las que no se ha observado ningún patrón ${ }^{92}$. En el caso de las drogas médicas, en general tienden a ser más consumidas por personas con mejor situación socioeconómica 49,100, aunque existen estudios que no encontraron variación en función de variables sociales ${ }^{92}$. En países industrializados el consumo de medicamentos psicotrópicos es más frecuente en los sujetos de mayor escolaridad, pero la dependencia se comporta en forma opuesta 140 .

Aunque en varias muestras de adolescentes se ha observado que los de clases superiores el consumo de drogas ilegales es más frecuente $122,124,125,126,127,128$, en otras investigaciones se reporta lo contrario $116,120,121,123$ o no se ha encontrado asociación 110,128. Un problema metodológico de estas investigaciones es que se estudian grupos de sustancias 119,121,124,125,126,127,130,132, lo cual no permite analizar diferencias entre ellas, pues en adultos se han vistos patrones consistentes (i.e., las drogas médicas son más frecuentes en estratos altos y las ilegales son más frecuentes en niveles bajos). De igual modo, cuando se ha analizado el consumo de drogas específicas por adolescentes se ha observado que las mejoras en la condición socioeconómica se han relacionado con mayor uso de drogas médicas 119,120,128, pero menor de inhalantes 119,128,131.

En los estudios sobre consumo de drogas en adolescentes ha sido frecuente compararlos de acuerdo al tipo de financiamiento de las escuelas (público versus privado) 116,120 o a las caracterís- 
ticas socioeconómicas de la zona donde se ubica la escuela donde estudian 114,115,128,129. Estos estudios pueden estar sesgados, ya que se excluyen a los individuos que no están matriculados en las instituciones educativas, los cuales por lo regular tienen peores condiciones socioeconómicas; además, usar al tipo de escuela como indicador socioeconómico tiene la desventaja de que no refleja adecuadamente la situación socioeconómica de los individuos (puede haber estudiantes de estrato alto en escuelas de zonas precarias y viceversa). Un sesgo más es que puede reflejar el ambiente escolar que, en sí mismo, puede ser un factor de riesgo para el consumo de drogas.

Conviene ahora comentar algunos aspectos metodológicos de los estudios revisados. En primer término, una proporción importante de los estudios revisados utilizaron muestras pequeñas por conveniencia o representativas de áreas geográficas reducidas, con lo cual se limita la posibilidad de extrapolar sus hallazgos a poblaciones más amplias; además, en los estudios en los que se entrevista a sujetos que acuden a instituciones de salud o educativas pueden existir sesgos de selección. Otra limitación de los estudios analizados es que son transversales, lo que impide establecer conclusiones en materia de causalidad. Desde la primera generación de estudios en epidemiología psiquiátrica surgió el debate respecto a si la relación inversa entre nivel socioeconómico y trastornos mentales (sin embargo, como se señalará más adelante, esta distribución no siempre ocurre) era explicada por la hipótesis de la causalidad social o por la de selección social 29,30,31,34. En la hipótesis de causalidad social se establece que la mayor prevalencia de trastornos mentales en los sujetos de estratos bajos se debe a que ellos se enfrentan a más eventos estresantes y cuentan con menos recursos para afrontarlos, mientras que la hipótesis de selección social establece que los trastornos mentales provocan que los sujetos que los padecen no se integren de forma exitosa al mercado laboral por lo que tienden a concentrarse en los estratos bajos. Los estudios realizados en América Latina no permiten esclarecer cuál de estas dos hipótesis es más factible de que ocurra, pues se basan en diseños transversales. Sin embargo, en estudios de cohorte realizados en países industrializados se ha mostrado que la selección social explica la relación negativa de la condición socioeconómica con esquizofrenia, trastornos de conducta y por déficit de atención; pero para la depresión, la ansiedad y el abuso de sustancias es más posible que opere la causalidad social 141,142,143.

Respecto a la medición de la estratificación, en algunos casos no se describe adecuadamente la construcción de clasificaciones de estrato o nivel socioeconómico 60,61,104, lo que limita la posibilidad de comparación entre estudios. La escolaridad de los entrevistados (en el caso de los adultos o del jefe de familia en los adolescentes) ha sido el indicador más utilizado en los estudios en América Latina, lo que probablemente se deba a que es una variable fácil de indagar y permite comparar entre distintas poblaciones. Menos estudios 40,44,47,50,52,54,65,66 evalúan la situación ocupacional (empleado versus desempleado-subempleo), la cual es relevante dada la estructura social de los países latinoamericanos, caracterizada por la elevada proporción de desempleo y empleo informal. Además, en ocasiones dicha variable no refleja la estratificación socioeconómica ya que, por ejemplo, en un grupo se incluyen a los estudiantes y las amas de casa, a quienes sería más conveniente clasificar de acuerdo a la ocupación del jefe de familia.

Con relación a las estrategias de análisis, en varios reportes, en lugar de comparar tasas de prevalencia entre los grupos socioeconómicos, se comparan las características sociodemográficas de los casos de trastornos mentales con las de la población general 40,60 o de los usuarios de una droga específica con las de usuarios en general o la población en general 106,131. Inclusive existen estudios en los que no se presentan medidas de significancia estadística. Estas deficiencias analíticas no permiten hacer una evaluación suficientemente objetiva de la asociación entre estratificación socioeconómica y enfermedad mental.

Un aspecto poco explorado es el posible papel del sexo como modificador de efecto, si bien en varios análisis el sexo es considerado un confusor que se ajusta en modelos de regresión. Con excepción del trabajo de Almeida-Filho et al. 57,96, no se han realizado análisis estratificados de acuerdo al sexo, lo cual es de relevancia, ya que la depresión y la ansiedad son más prevalentes en las mujeres mientras que en los varones son más altas las tasas de trastornos por consumo de sustancias 144. Las diferencias entre hombres y mujeres en las prevalencias de los trastornos específicos pueden provocar que las diferencias socioeconómicas en depresión y ansiedad se observen en mujeres, pero no en hombres (o por lo menos las diferencias sean más amplias en las primeras que en los últimos) 57; y que por el contrario, la estratificación socioeconómica se relacione con el consumo y abuso de sustancias en hombres, pero no en mujeres 96. En un estudio en Chile 53,54,55, en los hombres, pero no en las mujeres, la ocupación mantuvo relación con los trastornos mentales, mientras que lo contrario ocurrió con el ingreso y la escolaridad de los padres. En la población total del Distrito Federal la escolaridad no se relacionó claramente con la 
depresión y la distimia 62 , pero al hacer el análisis de hombres y mujeres por separado, en los primeros la escolaridad sólo se relacionó con la agorafobia, mientras que en las mujeres se asoció con agorafobia, agorafobia simple, fobia específica y fobia social 63; desafortunadamente en la primera publicación 62 no se realizó el análisis de cada sexo por separado.

Algunos autores de la epidemiología social plantean que para comprender los efectos de la inequidad social sobre la salud debe considerarse que los sujetos de estratos socioeconómicos bajos, debido a sus condiciones de vida, tienen mayor susceptibilidad para desarrollar diferentes patologías 145. Los estudios revisados no apoyan por completo esta premisa, pues si bien los adolescentes y adultos de estratos bajos presentan mayor riesgo de trastornos mentales o sintomatología relacionada con éstos y de dependencia al alcohol y ciertas drogas, también es cierto que las personas de estratos altos tienen más probabilidad de reportar consumo de alcohol y de drogas médicas.

Ya en nuestra época, los trabajos realizados en las últimas décadas indican que la relación entre las desigualdades socioeconómicas y las enfermedades mentales no ha desaparecido, pero existen posturas contradictorias respecto al papel de la pobreza, la ocupación, la educación y otras condiciones sociales sobre la salud mental. Algunas revisiones 32 fortalecen la idea de que las clases más desprotegidas se encuentran en peores condiciones que las más favorecidas, pero con matices con respecto a las propuestas que la medicina social realizaba hace treinta años 28 .

Desde que dieron inicio los estudios sobre desigualdad social y salud se ha insistido en el reto que representa la interpretación de sus resultados. Esto se debe, en primer lugar, al hecho de que los indicadores únicamente adquieren significado en el contexto específico en el que son recogidos. En segundo término debe reconocerse que la conducta de los individuos no puede separarse del contexto en el que se desenvuelven, pero siempre existe un cierto grado de autonomía individual que resulta muy difícil de evaluar. Por último, es necesario advertir que aunque ambos elementos - las conductas individuales y los contextos de desigualdad - se encuentran determinados por la estructura social, cada caso posee vías específicas para expresarse. Lo anterior significa que es posible indagar los mecanismos causales específicos que vinculan ciertos trastornos mentales con la desigualdad socioeconómica, pero cada situación investigada requiere de mecanismos explicativos elaborados específicamente para ella.

Para poder avanzar en este sentido se requiere enfrentar un problema que esta revisión ha hecho evidente: aunque cada vez existe mayor claridad en las formas de clasificación de los trastornos mentales, este no es el caso para la una medición de la desigualdad. No sólo no existe un indicador acabado que de cuenta de la desigualdad socioeconómica, sino que ni siquiera existe consenso sobre el uso de los indicadores que hasta ahora se han utilizado. La razón, de acuerdo con Regidor 13, es sencilla: todavía se desconoce con exactitud qué se está midiendo. En efecto, la utilización idónea de un concepto implica su definición teórica inicial y, con base en ella, su operativización en componentes susceptibles de ser medidos y usados para realizar comparaciones. Es evidente que la región latinoamericana adolece de los mismos problemas teóricos y prácticos que se presentan en otras regiones del mundo: la medición de las desigualdades y la explicación precisa de su impacto en la salud mental aún requiere un intenso trabajo conceptual y empírico.

Debe señalarse, finalmente, que existen temas que han sido explorados insuficientemente, entre los que se encuentran: la relación entre posición socioeconómica y salud mental en adolescentes, las diferencias entre estratos en la recuperación o recaída de trastornos mentales y abuso y dependencia de sustancias, y la distribución de la comorbilidad en función de factores socioeconómicos específicos, como el género y las moldes culturales. 


\section{Resumen}

El objetivo fue analizar la producción científica realizada en América Latina que indaga el impacto de la condición socioeconómica sobre los trastornos mentales y el uso o dependencia a sustancias. Se revisaron bases de datos internacionales y regionales. En la mayoría de los estudios se ha observado que en adultos y adolescentes pertenecer a estratos socioeconómicos bajos se asocia con mayor riesgo de trastornos mentales. El consumo de alcohol es mayor entre las personas con mejores condiciones socioeconómicas; en el caso del abuso y la dependencia al alcohol se observa una tendencia opuesta, aunque la evidencia para los últimos es menos contundente. En adolescentes el tabaquismo es más frecuente entre los que provienen de hogares con mejores condiciones socioeconómicas; sin embargo, entre los adultos la mayor probabilidad se encuentra entre los de peor situación económica. En adultos el consumo de drogas ilegales es más frecuente entre las personas de nivel socioeconómico inferior. Las drogas médicas tienden a ser más consumidas por adultos y adolescentes con mejor situación socioeconómica. El uso de solventes es más frecuente entre adolescentes de estratos bajos.

Trastornos Relacionados con Sustancias; Estrés Psicológico; Trastornos Mentales; Factores Socioeconómicos

\section{Referencias}

1. Levav I, Lima BR, Somoza M, Kramer M, González R. Salud mental para todos en América Latina el Caribe. Bases epidemiológicas para la acción. Bol Oficina Sanit Panam 1989; 107:196-219.

2. Murray CJ, Lopez AD. Global mortality, disability, and the contribution of risk factors: Global Burden of Disease Study. Lancet 1997; 349:1436-42.

3. Üstün TB, Ayuso-Mateos JL, Chatterji S, Mathers C, Murray CJL. Global burden of depressive disorders in the year 2000. Br J Psychiatry 2004; 184:386-92.

4. Organización Panamericana de la Salud. Iniciativa regional de datos básicos en salud. Sistema generador de tablas. http://www.paho.org/Spanish/ SHA/coredata/tabulator/newTabulator.htm (accedido el 28/Mar/2005).

5. Alleyne GAO, Castillo-Salgado C, Schneider MC, Loyola E, Vidaurre M. Overview of social inequalities in health in the Region of the Americas using various methodological approaches. Rev Panam Salud Pública 2002; 12:388-97.

\section{Colaboradores}

Los autores participaron de cada una de las fases de preparación del manuscrito igualmente.
6. Loyola E, Castillo-Salgado C, Nájera-Aguilar P, Vidaurre M, Mujica OJ, Martínez-Piedra R. Los sistemas de información geográfica como herramienta para monitorear las desigualdades de salud. Rev Panam Salud Pública 2002; 12:415-28.

7. Milanovic B, Yitzhaki S. Decomposing world income distribution. Washington DC: World Bank; 2001. (Policy Research Working Paper, 2562).

8. Comisión Económica para América Latina y el Caribe. Panorama social de la América Latina, 20002001. Santiago de Chile: Comisión Económica para América Latina y el Caribe; 2001.

9. Rosen G. De la policía médica a la medicina social. México DF: Siglo XXI Editores; 1985.

10. Ramazzini B. Las enfermedades de los trabajadores. De mortis artificum diatriba. México DF: Editorial Porrúa/Universidad Autónoma Metropolitana Xochimilco; 2000.

11. Sigerist H. Hitos en la historia de la salud pública. México DF: Siglo XXI Editores; 1981. 
12. Caponi S. Entre miasmas y microbios: la vivienda popular higienizada. Cad Saúde Pública 2002; 18:1665-74.

13. Regidor E, Gutiérrez-Fisac JL, Rodríguez C. Diferencias y desigualdades en salud en España. Madrid: Ediciones Díaz de Santos; 1994.

14. Townsend P, Davidson N. Inequalities in health: the Black Report. Harmondsworth: Penguin; 1982.

15. Krieger N, Williams DR, Moss NE. Measuring social class in US public health research: concepts, methodologies, and guidelines. Annu Rev Public Health 1997; 18:341-78.

16. Breilh J. Economía, medicina y política. Quito: Universidad Central del Ecuador; 1979.

17. Bronfman M, Tuirán R. La desigualdad social ante la muerte: clases sociales y mortalidad en la niñez. Cuad Méd Soc 1984; (29-30):53-75.

18. Wolhfarth T, van der Brink W. Social class and substance use disorders: the value of social class as distinct from socioeconomic status. Soc Sci Med 1998; 47:51-8.

19. Muntaner C, Borell C, Benach J, Pasarín MI, Fernández E. The associations of social class and social stratification with patterns of general and mental health in a Spanish population. Int J Epidemiol 2003; 32:950-8.

20. Rawls J. La justicia como equidad: una reformulación. Barcelona: Paidós; 2002.

21. Sen A. La desigualdad económica. México DF: Fondo de Cultura Económica; 2001.

22. Whitehead M. The concepts and principles of equity and health. Copenhagen: World Health Organization Regional Office for Europe; 1990.

23. Marmot M, Kogevinas M, Elston MA. Social/economic status and disease. Annu Rev Public Health 1987; 8:111-35.

24. Daniels N, Kennedy B, Kawachi I. Is inequality bad for our health? Boston: Beacon Press Books; 2000.

25. Krieger N. Glosario de epidemiología social. Rev Panam Salud Pública 2002; 11:480-90.

26. Almeida-Filho N. Inequalities in health based on living conditions. Washington DC: Pan American Health Organization; 1999.

27. Spinelli H, Urquía H, Bargalló ML, Alazraqui M. Equidad en salud: teoría y praxis. In: Spinelli H, compilador. Salud colectiva. Buenos Aires: Lugar Editorial; 2004. p. 261-2.

28. Bastide R. Sociología de las enfermedades mentales. México DF: Siglo Veintiuno Editores; 1979.

29. Dohrenwend BP, Dohrenwend BS. Perspectives on the past and future of psychiatric epidemiology. Am J Public Health 1982; 72:1271-9.

30. Dohrenwend BP. Socioeconomic status (SES) and psychiatric disorders. Soc Psychiatry Psychiatr Epidemiol 1990; 25:41-7.

31. Dohrenwend BP, Schwartz S. Socioeconomic status and psychiatric disorders. Curr Opin Psychiatry 1995; 8:138-41.

32. Fryers T, Melzer D, Jenkins R. Social inequalities and the common mental disorders. Soc Pschiatry Psychiatr Epidemiol 2003; 38:229-37.

33. Galea S, Nandi A, Vlahov D. The social epidemiology of substance use. Epidemiol Rev 2004; 26:36-52.

34. Muntaner C, Eaton WE, Miech R, O'Campo P. Socioeconomic position and major mental disorders. Epidemiol Rev 2004; 26:53-62.
35. Almeida-Filho N. Social epidemiology of mental disorders. A review of Latin-American studies. Acta Psychiatr Scand 1987; 75:1-10.

36. Patel V, Araya R, De Lima M, Ludermir A, Todd C. Women, poverty and common mental disorders in four restructuring societies. Soc Sci Med 1999; 49:1461-71.

37. Patel V, Kleinman A. Poverty and common mental disorders in developing countries. Bull World Health Organ 2003; 81:609-15.

38. Borges G, Rosovsky H, Gil A, Pelcastre B, López JL. Análisis de casos y controles de los intentos de suicidio en una muestra de servicios de urgencia. Anales del Instituto Mexicano de Psiquiatría 1993; 4:198-203.

39. Botega NJ, Berti M, Bosco H, Dalgalarrondo P, Marín-León L. Suicidal behavior in the community: Prevalence and factors associated with suicidal ideation. Rev Bras Psiquiatr 2005; 27:45-53.

40. Medina-Mora ME, Rascón M, Tapia R, Mariño M, Juárez F, Villatoro J, et al. Trastornos emocionales en población urbana mexicana: resultados de un estudio nacional. Anales del Instituto Mexicano de Psiquiatría 1992, 3:48-55.

41. Jadresic E, Araya R. Prevalencia de depresión postparto y factores asociados en Santiago, Chile. Rev Méd Chile 1995; 123:694-9.

42. Villanueva LA, Pérez-Fajardo MM, Iglesias LF. Factores sociodemográficos asociados a depresión en adolescentes embarazadas. Ginecol Obstetr Méx 2000; 68:143-7.

43. Illanes E, Bustos L, Lagos X, Navarro N, Muñoz S. Factores asociados a síntomas depresivos y síntomas somáticos en mujeres climatéricas de la ciudad de Temuco. Rev Méd Chile 2002; 130:885-91.

44. Gazalle FK, De Lima MS, Tavares BF, Hallal PC. Sintomas depressivos e fatores associados em população idosa no Sul do Brasil. Rev Saúde Pública 2004; 38:365-71.

45. Di Marco G. Prevalencia de desórdenes mentales en el área metropolitana de la República Argentina. Acta Psiquiatr Psicol Am Lat 1982; 28:93-102.

46. Penayo U, Caldera T, Jacobsson L. Prevalencia de trastornos mentales en adultos de Subtiava, León, Nicaragua. Bol Oficina Sanit Panam 1992; 113:13748.

47. Freire ES, Almeida Filho N, Mari JJ, Rodrigues L. Minor psychiatric morbidity and internal migration in Brazil. Soc Psychiatry Psychiatr Epidemiol 1996; 31:173-9.

48. Freire E, Almeida-Filho N, Mari JJ. Fatores de risco para morbidade psiquiátrica menor: resultados de um estudo transversal em três áreas urbanas no Brasil. Rev Psiquaitr Clin (São Paulo) 1999; 26:24656.

49. Lima MS, Soares BGO, Mari JJ. Saúde e doença mental em Pelotas, RS: dados de um estudo populacional. Rev Psiquiatr Clin (São Paulo) 1999; 26:225-35.

50. Blue I. Individual and contextual effects on mental health status in São Paulo, Brazil. Rev Bras Psiquiatr 2000; 22:116-23.

51. Ludermir AB, Lewis G. Links between social class and common mental disorders in Northeast Brazil. Soc Psychiatry Psychiatr Epidemiol 2001; 36:101-7. 
52. Ludermir AB, Melo DA. Condições de vida e estrutura ocupacional associadas a transtornos mentais comuns. Rev Saúde Pública 2002; 36:213-21.

53. Araya R, Rojas G, Fritsch R. Depresión y género en Santiago de Chile. Acta Psiquiatr Psicol Am Lat 2000; 46:325-35.

54. Araya R, Rojas G, Fritsch R, Acuña J, Lewis G. Santiago Mental Disorders Survey: prevalence and risk factors. Br J Psychiatry 2001; 178:228-33.

55. Araya R, Lewis G, Rojas G, Fritsch R. Education and income: which is more important for mental health? J Epidemiol Community Health 2003; 57:501-5.

56. Almeida-Filho N, Mari JJ, Coutinho ESF. Migração, inserção produtiva e saúde mental na modernidade tardia: novas evidências do estudo multicêntrico de morbidade psiquiátrica em áreas metropolitanas brasileiras. Rev Psiquiatr Clin (São Paulo) 1999; 26:236-45

57. Almeida-Filho N, Lessa I, Megalhães L, Araújo MJ, Aquino E, Sherman A, et al. Social inequality and depressive disorders in Bahia, Brazil: interactions of gender, ethnicity, and social class. Soc Sci Med 2004; 59:1339-53.

58. Maia LC, Durante AMG, Ramos LR. Prevalência de transtornos mentais em área urbana no norte de Minas Gerais, Brasil. Rev Saúde Pública 2004; 38:650-6.

59. Kac G, Silveira EA, Costa L, Mari JJ. Fatores relacionados à prevalência de morbidades psiquiátricas menores em mulheres selecionadas em um Centro de Saúde no Rio de Janeiro, Brasil. Cad Saúde Pública 2006; 22:999-1007.

60. Berenzon S, Medina-Mora ME, López EK, González J. Prevalencia de trastornos mentales y variables asociadas en cuatro comunidades del sur de la ciudad de México. Revista Mexicana de Psicología 1998; 15:177-85.

61. Berenzon S, González-Forteza C, Medina-Mora ME. Asociación entre trastornos depresivos y fóbicos con ideación e intento suicida en mujeres de comunidades urbanas pobres. Revista Mexicana Psicología 2000; 17:55-63.

62. Caraveo-Anduaga J, Colmenares E, Saldívar E, Saldívar G. Estudio clínico-epidemiológico de los trastornos depresivos. Salud Ment 1999; 22:7-17.

63. Caraveo-Anduaga J, Colmenares E. Prevalencia de los trastornos de ansiedad fóbica en la población adulta de la ciudad de México. Salud Ment 2000; 23:10-9.

64. Andrade L, Walters EE, Gentil V, Laurenti R. Prevalence of ICD-10 mental disorders in a catchment area in the city of São Paulo, Brazil. Soc Psychiatry Psychiatr Epidemiol 2002; 37:316-25.

65. Vorcaro CMR, Lima-Costa MFF, Barreto MS, Uchoa E. Unexpected high prevalence of 1-month depression in a small Brazilian community: the Bambui Study. Acta Psychiatr Scand 2001; 104:257-63.

66. Vocaro CMR, Rocha FL, Ochoa E, Lima-Costa MF. The burden of social phobia in a Brazilian community and its relationship with socioeconomic circumstances, health status and use of health services: the Bambuí Study. Int J Soc Psychiatry 2004; 50:216-26.
67. Gómez-Restrepo C, Bohórquez A, Pinto D, Gil JFA, Rondon M, Díaz-Granados N. Prevalencia de depresión y factores asociados con ella en la población colombiana. Rev Panam Salud Pública 2004; 16:378-86.

68. Vicente B, Rioseco P, Saldivia S. Chilean study on the prevalence of psychiatric disorders. Rev Méd Chile 2002; 130:527-36.

69. Vicente V, Kohn R, Rioseco P, Valdivia S, Baker C, Torres S. Population prevalence of psychiatric disorders in Chile: 6-month and 1-month rates. Br J Psychiatry 2004; 184:299-305.

70. Canino GJ, Bird HR, Shrout PE, Rubio-Stipec M, Bravo M, Martínez R, et al. The prevalence of specific psychiatric disorders in Puerto Rico. Arch Gen Psychiatry 1987; 44:727-35.

71. Monreal M, Montoya C. Desigualdades en salud mental. Chile 1997-2001. Cuad Méd Soc 2004; 44:91-101.

72. Vicente B, Rioseco P, Vielma M, Boggiano G, Silva L, Saldivia S. El nivel socio-económico como factor de riesgo del enfermar psiquiátrico. Rev Psiquiatr Salud Ment 2000; 17:98-108.

73. Mondragón L, Saltijeral AT, Bimbela A, Borges G. La ideación suicida y su relación con la desesperanza, el abuso de drogas y alcohol. Salud Ment 1998; 21:20-7.

74. Mondragón L, Monroy Z, Medina-Mora ME, Borges G. Eventos de la vida, depresión, consumo de alcohol e ideación suicida en una muestra de servicios de urgencias: un enfoque de género. Revista Mexicana de Psicología 2003; 20:225-35.

75. Gómez-Restrepo C, Rodríguez N, Bohórquez A, Diaz N, Ospina MB, Fernández C. Factores asociados al intento de suicidio en la población colombiana. Rev Colomb Psiquiatr 2002; 31:271-86.

76. Marin-León L, Barros MBA. Mortes por suicídios: diferenças de gênero e nível socioeconômico. Rev Saúde Pública 2003; 37:357-63.

77. Pallini G, Rioseco P, Fasce M, Ebner D. Análisis epidemiológico de los suicidios registrados en el Servicio de Salud Concepción - Arauco (19941998). Psiquiatr Salud Ment 2003; 20:194-8.

78. Rapeli CB, Botega NJ. Tentativas de suicídio envolvendo risco de vida: internações em um hospital geral. J Bras Psiquiatr 1998; 47:157-62.

79. Rivera C. Análisis criminológico del suicidio en el Distrito Federal. Psiquiatría (Méx) 1998; 14:33-44.

80. Ortiz-Maldonado E, Cornelio-Dionisio A, ReynosoOrnelas C, Toledo-Hernández TJ, Torre-Trujillo PC. Factores de riesgo suicida en los municipios de Cárdenas, Comalcalco y Centro del estado de Tabasco. Salud en Tabasco 2002; 8:107-14.

81. Lucero R, Villalva L. Caracterización clínica y epidemiológica de los suicidios en Montevideo y de los intentos de autoeliminación (IAE) en el Hospital de Clínicas en el periodo abril 2000-abril 2001. Rev Psiquiatr Urug 2003; 67:5-20.

82. Castelli P, Serrano C. Caracterización del paciente que acude por intento suicida a un hospital general. Acta Psiquiatr Psicol Am Lat 1998; 44:268-77.

83. García ME, Tapia A. Epidemiología del suicidio en el estado de Tabasco en el periodo 1979-1988. Salud Ment 1990; 13:29-34. 
84. Corlay IS, Vázquez MF, Ariza A, Ruiz LG. Intentos de suicidio en el Hospital de Especialidades CM La Raza. Análisis de casos atendidos de 1986 a 1988. Rev Méd IMSS 1990; 28:269-73.

85. Juárez F, Medina-Mora ME, Berenzon S, Villatoro JA, Carreño S, López EK, et al. Antisocial behaviour: its relations selected sociodemographic variables and alcohol and drug use among Mexican students. Subst Use Misuse 1998; 33:1437-59.

86. Juárez-Aragón G, Castañón-González JA, PérezMorales AJ, Montoya MA. Características clínicas y epidemiológicas de intoxicaciones graves en una población adulta que ingresa a una unidad de cuidados intensivos. Gac Méd Méx 1999; 165:669-75.

87. Vázquez-Segovia LA, Sesma-Vázquez S, Hernández-Ávila M. El consumo de tabaco en los hogares en México: resultados de la encuesta de ingresos y gastos de los hogares, 1984-2000. Salud Pública Méx 2002; 44 Suppl 1:S76-81.

88. Sesma-Vázquez S, Campuzano JC, Carreón-Rodríguez VG, Knaul F, López-Antuñano FJ, HernándezÁvila M. El comportamiento de la demanda de tabaco en México: 1992-1998. Salud Pública Méx 2002;44 supl 1:S82-S92.

89. Lolio CAD, Souza JMPD, Santo AH, Buchalla CM. Prevalência de tabagismo em localidade urbana da região sudeste do Brasil. Rev Saude Pública $1993 ; 27: 262-5$.

90. Moreira LB, Fuchs FD, Moraes RS, Bredemeier M, Cardozo S. Prevalência de tabagismo e fatores associados em área metropolitana da região Sul do Brasil. Rev Saúde Pública 1995; 29:46-51.

91. Moreira LB, Fuchs FD, Moraes RS, Bredemeier M, Cardozo S, Fuchs SC, et al. Alcoholic beverages consumption and associated factors in Porto Alegre, a southern Brazilian City: a population based survey. J Stud Alcohol 1996; 57:253-9.

92. Fuentealba R, Cumsille F, Araneda AC, Molina C. Consumo de drogas lícitas e ilícitas en Chile: resultados del estudio de 1998 y comparación con los estudios de 1994 y 1996. Rev Panam Salud Pública 2000; 7:79-87.

93. Kroeff LR, Mengue SS, Schmidt MI, Duncan BB, Favaretto ALF, Nucci LB. Fatores associados ao fumo em gestantes avaliadas em cidades brasileiras. Rev Saúde Pública 2004; 38:261-7.

94. Almeida LM, Countinho ESF. Prevalência de consumo de bebidas alcoólicas e de alcoolismo em uma região metropolitana do Brasil. Rev Saúde Pública 1993; 27:23-9.

95. Bejarano J, Carvajal H. El consumo e drogas en la mujer costarricense. Revista de Ciencias Sociales 1994; (65):43-55.

96. Almeida-Filho N, Lessa I, Magalhães L, Araújo MJ, Aquino E, Kawachi I, et al. Alcohol drinking patterns by gender, ethnicity, and social class in Bahia, Brazil. Rev Saúde Pública 2004; 38:45-54.

97. Villatoro-Velázquez JA, Medina-Mora ME, LópezLugo EK, Juárez FL, Rivera E, Fleiz C. La dependencia y los problemas asociados al consumo de drogas en México: resultados de la Encuesta Nacional de Adicciones. Salud Ment 1996; 19:1-6.

98. Medina E, Kaempffer AM, Cornejo E, Hernández E. El beber problema en la población de Santiago. Rev Psiquiatr (Santiago de Chile) 1996; 12:67-73.
99. Bejarano J, Carvajal H, Lee LS. Alcohol y alcoholismo en la sociedad costarricense. Revista de Ciencias Sociales 1997; (77):9-20.

100.Míguez H. Consumo de sustancias psicoactivas en Argentina. Acta Psiquiatr Psicol Am Lat 2000; 46:247-54.

101.Mendoza-Sassi RA, Béria JU. Prevalence of alcohol use disorder and associated factors: a populationbased study using AUDIT in southern Brazil. Addiction 2003; 98:799-804.

102. Rosovsky H, Casanova L, Pérez C. Las características de los grupos y de los miembros de Alcohólicos Anónimos. Anales del Instituto Mexicano de Psiquiatría 1991; 2:138-42.

103.Bejarano J, Carvajal H. Abuso de drogas y conducta delictiva. Revista de Ciencias Sociales 1993; (60):51-62.

104.Benassini O, Guzmán AC. Patrones de abuso en consumidores de drogas: reporte comparativo a 6 años. Psiquiatría (Méx) 1994; 10:42-7.

105.Silva EA, Ferri CP, Formigoni MLOS. Situações de recaída em pacientes dependentes de álcool e outras drogas durante o tratamento: um estudo preliminar. J Bras Psiquiatr 1995; 44:311-5.

106.Unikel C, Galván J, Soriano A, Villatoro J, Ortíz A, Rodríguez E. Evolución del consumo de cocaína en México y su presencia entre las clases menos favorecidas. Salud Ment 1998; 21:29-34.

107.Cravioto P, De La Rosa B, Tapia-Conyer R. Riesgo de consumo de drogas ilegales en población económicamente activa en México. Psiquis 2001; 10:17-26.

108.López EK, Medina-Mora ME, Villatoro JA, Juárez F, Carreño S, Berenzon S, et al. La relación entre la ideación suicida y el abuso de sustancias tóxicas. Resultados de una encuesta en la población estudiantil. Salud Ment 1995; 18:25-32.

109.González-Forteza C, Villatoro J, Pick S, Collado ME. El estrés psicosocial y su relación con las respuestas de enfrentamiento y el malestar emocional en una muestra representativa de adolescentes al sur de la ciudad de México: análisis según el nivel socioeconómico. Salud Ment 1998; 21:37-45.

110.McWhriter PC, Florenzano R, Florenzano M. Correlatos psicosociales de las farmacodependencias: resultados de un estudio en Santiago de Chile. Rev Psiquiatr (Santiago de Chile) 1998; 15:10-22.

111.Miranda CA, Gutiérrez JC, Bernal F, Andrés C. Prevalencia de depresión en estudiantes de medicina de la U. del Valle. Rev Colomb Psiquiatr 2000; 29:251-60.

112.Bralio S, Seguel X, Montenegro H. Prevalencia de trastornos psíquicos en la población escolar de Santiago de Chile. Acta Psiquiatr Psicol Am Lat 1987; 33:316-25.

113.Pineda D, Ardila A, Rosselli M, Arias BE, Henao GC, Gómez LE, et al. Prevalence of attention-deficit/ hyperactivity disorder symptoms in 4- to 17 yearold children in the general population. J Abnorm Child Psychol 1999; 27:455-62.

114.Maya MA, García G. Estudio epidemiológico sobre el uso de alcohol en población joven de 14 a 18 años. Salud Pública Méx 1986; 28:371-9. 
115.Caroma AE, Rodríguez G, Ardiles J, Águila M, Gallardo G, Santander D. Factores socioeconómicos e ingestión de alcohol en estudiantes secundarios. Rev Méd Chile 1988; 114:474-82.

116.Bergonzoli G, Rico O, Ramírez A, Paz MI, Ramírez $\mathrm{J}$, Rivas JC, et al. Uso de drogas entre estudiantes de Cali, Colombia. Bol Oficina Sanit Panam 1989; 106:23-30.

117.Badilla B, Barquero M. Prevalencia del uso de estimulantes en los estudiantes de medicina de la Universidad de Costa Rica. Revista de Ciencias Sociales 1993; (61):121-9.

118.Oderich GSC, Pechansky F, Tatsch FF, Cavazzola LT, Boeno RL, Menegaz F. Consumo de bebidas alcoólicas em alunos de escolas públicas de Porto Alegre, RS. Rev AMRIGS 1995; 39:229-36.

119.Medina-Mora ME, Villtoro JA, López EK, Berenzon $\mathrm{S}$, Carreño S, Juárez F. Los factores que se relacionan con el inicio, el uso continuado y el abuso de sustancias psicoactivas en adolescentes mexicanos. Gac Méd Méx 1995; 131:383-93.

120.Sandí L, Díaz A. Consumo de drogas y problemas asociados en estudiantes costarricenses. Revista de Ciencias Sociales 1996; (73-74):17-25.

121.Caballero R, Madrigal E, Hidalgo A, Villaseñor, A. El consumo de tabaco, alcohol y drogas ilegales, en los adolescentes de diferentes estratos socioeconómicos de Guadalajara. Salud Ment 1999; 22:1-8.

122.Queiroz S, Scivoletto S, Souza MM, Strassman PG, Gattaz WF. Uso de drogas entre estudantes de uma escola pública de São Paulo. Rev Psiquaitr Clin (São Paulo) 2001; 28:176-82.

123.Baus J, Kupek E, Pires M. Prevalência e fatores de risco relacionados ao uso de drogas entre escolares. Rev Saúde Pública 2002; 36:40-6.

124.Soldera M, Dalgalarrondo P, Corrêa Filho HR, Silva CA. Heavy alcohol use among elementary and high-school students in downtown and outskirts of Campinas City - São Paulo: prevalence and related factors. Rev Bras Psiquiatr 2004; 26:174-9.

125.Soldera M, Dalgalarrondo P, Corrêa Filho HR, Silva CA. Uso de drogas psicotrópicas por estudantes: prevalência e fatores sociais associados. Rev Saúde Pública 2004; 38:277-83.

126.Herrera-Vázquez M, Wagner FA, Velasco-Mondragón E, Borges G, Lazcano-Ponce E. Inicio en el consumo de alcohol y tabaco y transición a otras drogas en estudiantes de Morelos, México. Salud Pública Méx 2004; 46:132-40.

127. Muza MM, Bettiol H, Muchillo G, Barberi MA. Consumo de substâncias psicoactivas por adolescentes escolares de Riverão Preto, SP (Brasil). Rev Saúde Pública 1997; 31:21-9.

128.Castro ME, Maya MA, Aguilar MA. Consumo de sustancias tóxicas y tabaco entre población estudiantil de 14-18 años. Salud Pública Méx 1982; 24:565-74.

129.Velásquez MG, López C, Borges G, Pelcastre B. Prevalencia y consumo de tabaco de una población estudiantil de siete escuelas secundarias del D.F. Salud Ment 1992; 15:42-7.

130.Souza DPO, Martins DT. O perfil epidemiológico do uso de drogas entre estudantes de lo e 2 o graus da rede estadual de ensino de Cuiabá, Brasil, 1995. Cad Saúde Pública 1998; 14:391-400.
131.Berenzon S, López EK, Medina-Mora ME, Villatoro, Juárez F, Carreño S, et al. Relación entre consumo de inhalantes y actos antisociales en una muestra de estudiantes del Distrito Federal. Anales del Instituto Mexicano de Psiquiatría 1994; 5:94-9.

132.Medina-Mora ME, Rojas E. Mujer, pobreza y adicciones. Perinatol Reprod Hum 2003; 17:230-44.

133.Kessler RC, McGonagke KA, Zhao S, Nelson CB, Hughes M, Eshleman S, et al. Lifetime and 12month prevalence of DSM-III-R psychiatric disorders in the United States. Arch Gen Psychiatry 1994; 51:8-19.

134.Blazer DG, Kessler RC, McGonagle KA, Swartz MS. The prevalence and distribution of major depression in a national community sample: the National Comorbidity Survey. Am J Psychiatry 1994; 151:979-86.

135.Liberatos P, Bruce GL, Kelsey JL. The measure of social class in epidemiology. Epidemiol Rev 1988; 10:87-121.

136.Bosma H, van de Mheen HD, Mackenbach JP. Social class in childhood and general health in adulthood: questionnaire study of contribution of psychological attributes. BMJ 1999; 318:18-22.

137.Weich S, Lewis G, Jenkins SP. Income inequality and the prevalence of common mental disorders in Britain. Br J Psychiatry 2001; 178:222-7.

138.Muntaner C, Eaton WW, Diala C, Kessler RC, Sorlie PD. Social class, assets, organizational control and the prevalence of common groups of psychiatric disorders. Soc Sci Med 1998; 47:2043-53.

139.Borges G, Medina-Mora ME, López-Moreno S. El papel de la epidemiología en la investigación de los trastornos mentales. Salud Pública Méx 2004; 46:451-63.

140.Warner LA, Kessler RC, Hughes M, Anthony JC, Nelson CB. Prevalence and correlates of drug use and dependence in the United States. Results from the National Comorbidity Survey. Arch Gen Psychiatry 1995; 52:219-29.

141.Dohrenwend BP, Levav I, Patrick S, Schwartz S, Naveh G, Link B, et al. Socioeconomic status and psychiatric disorders: the causation-selection issue. Science 1992; 255:946-52.

142.Link BG, Clare M, Dohrenwend BP. Socioeconomic status and depression: the role of occupations involving direction, control and planning. Am J Sociol 1993; 98:1351-87.

143.Miech RA, Caspi A, Moffitt TE, Entner BR, Silva P. Low socioeconomic status and mental disorders: a longitudinal study of selection and causation during young adulthood. Am J Sociol 1999; 104:1096131.

144.Granados JA, Ortiz-Hernández L. Patrones de daños a la salud mental: psicopatología y diferencias de género. Salud Ment 2003; 26:42-50.

145.Ortiz-Hernández L. Contribuciones de la epidemiología social a la comprensión de las condiciones de salud de las poblaciones. Salud Probl 2003; 8:17-26.

Recibido el 28/Jun/2006

Aprobado 18/Ene/2007 